\title{
A Rare Constrictive Pericarditis with Complete Separation between the Visceral and Parietal Pericardium: A Case Report
}

\author{
Qianhui Sun, ${ }^{1}$ Liang Ma, MD, ${ }^{2}$ Peng Teng, $\mathrm{MD}^{2}$ \\ ${ }^{1}$ Department of Surgical Intensive Care Unit, The First Affiliated Hospital, College of Medicine, Zhejiang University, Zhejiang \\ University, Hangzhou, Zhejiang Province, P.R. China; ${ }^{2}$ Department of Cardiovascular Surgery, The First Affiliated Hospital, College \\ of Medicine, Zhejiang University, Zhejiang University, Hangzhou, Zhejiang Province, P.R. China
}

\section{ABSTRACT}

Background: Pericarditis is the most common form of pericardial disease, while constrictive pericarditis is challenging in diagnosis and is easily overlooked.

Case report: A 30-year-old female presented with abdominal distension and mild lower extremity edema for 3 months. The patient was initially suspected of having cirrhosis caused by Wilson Disease. Following liver biopsy and multiple investigation, thickened, calcified pericardium was detected by echocardiography and chest computed tomography. The patient was finally diagnosed with chronic constrictive pericarditis and received pericardiectomy. Intraoperatively, we found that the heart was entirely constricted by the thickened and calcified visceral pericardium, which was completely separated from the parietal pericardium. The patient received successful pericardiectomy and had relief of symptoms after surgery.

Conclusion: Patients with constrictive pericarditis may present with symptoms similar to that of chronic liver diseases, which makes it difficult and complicated for diagnosis. This case highlights the importance of comprehensive preoperative evaluation and maintaining clinical suspicion of pericarditis in patients with features of elevated systemic venous pressure. In addition, constrictive pericarditis with complete separation between visceral and parietal pericardium has seldom been reported.

\section{INTRODUCTION}

Constrictive pericarditis (CP) is caused by thickened and inelastic pericardium, and is commonly manifested with fibrous scarring and adhesion in both the visceral and parietal

Received April 17, 2020; received in revised form fune 11, 2020; accepted fune $11,2020$.

This work was supported by grants from Zhejiang Medical and Health Science and Technology Projects of China [Project number: 2018249646].

Peng Teng, MD, Department of Cardiovascular Surgery, The First Affiliated Hospital, College of Medicine, Zhejiang University, \#79 Qingchun Road, Hangzhou, Zhejiang Province, P.R. China, 310003; +86-0571-87236841; fax: +86-0571-87236843 (e-mail: tengpeng@zju.edu.cn). pericardium. It restricts and impairs the diastolic filling of the heart. Tuberculosis accounts for nearly half of cases, while cardiac surgery and chest radiation [Wei 2019] became two of the main causes in recent years. Pericardiectomy is the only definitive treatment option for symptomatic patients with chronic CP. However, patients with late stage CP are at the highest risk for surgery. Thus, timely diagnosis and subsequent surgical treatment is important. Herein, we report a 30-year-old female who presented with abdominal distension and mild lower extremity edema, which was initially suspected to be cirrhosis caused by Wilson Disease but was finally diagnosed as CP. The patient presented with rare intraoperative findings of complete separation between visceral and parietal pericardium and received successful pericardiectomy. To our best knowledge, constrictive pericarditis with complete separation between visceral and parietal pericardium has seldom been reported.
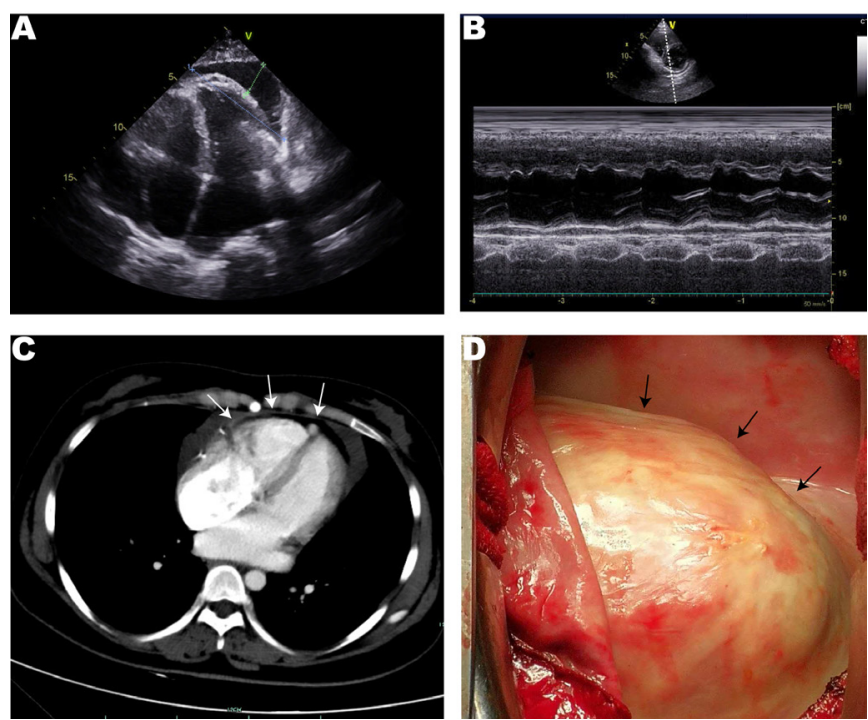

A, TTE showed mild pericardial effusion (about $8.1 \times 2.3 \mathrm{~cm}$ ), biatrial enlargement (left atrium was $66 \times 55 \mathrm{~mm}$, right atrium was $57 \times 44 \mathrm{~mm}$ ), and thickened lateral pericardium (about $6 \mathrm{~mm}$ ); B, M-mode echocardiography showed paradoxical interventricular septum motion; C, Chest CT showed the thickened visceral pericardium (arrow) and mild deformed ventricles; D, Intraoperative photograph showed the complete separation between the visceral (arrow) and parietal pericardium.TTE indicates transthoracic echocardiography; CT, computed tomography. 
CASE REPORT

A 30-year-old Chinese female with no history of tuberculosis, radiation, chest trauma or cardiac surgery was admitted to our hospital because of abdominal distension and lower extremity edema for 3 months. Her history of viral infection was not clear, as only several times of having a cold could be reported. Physical examination revealed blood pressure 103/78 $\mathrm{mmHg}$, pulse rate $92 / \mathrm{min}$ and regular, elevated jugular venous pressure, hepatomegaly, negative shifting dullness, and mild pitting edema in the lower extremity. The heart sounds were distant; no friction rub was noticed over the heart. Routine blood test showed normal blood cell count, and a liver function test showed normal aspartate transaminase, normal alanine transaminase, normal alkaline phosphatase, and elevated gamma-glutamyl transferase (61 IU/L). All the autoimmune antibody tests were negative. Her serum ceruloplasmin was $15.1 \mathrm{mg} / \mathrm{dL}$, which was under the normal range (22.0-58.0 $\mathrm{mg} / \mathrm{dL}$ ). No obvious abnormality was detected in the other laboratory tests. Electrocardiogram showed normal sinus rhythm without ST-segment elevation. Abdominal computed tomography (CT) suggested congestive liver cirrhosis, splenomegaly, edema of gallbladder wall, and mild ascites. Based on her decreased serum ceruloplasmin and liver cirrhosis, the patient was initially suspected as having Wilson Disease and underwent liver biopsy. Further assessment of transthoracic echocardiography (TTE) revealed normal mitral inflow E/A ratio (mitral E\&A velocity: 79 and $41 \mathrm{~cm} / \mathrm{s}$ ), normal left ventricular systolic function (EF: 61\%), bi-atrial enlargement (left \& right atrium: $66 \times 55$ and $57 \times 44 \mathrm{~mm}$ ), thickened pericardium (about $6 \mathrm{~mm}$ ), mild pericardial effusion (0.5-1 $\mathrm{cm})$ (Figure, A), dilated inferior vena cava $(24 \mathrm{~mm})$, paradoxical interventricular septum motion (Figure, B), and expiratory diastolic reversal in hepatic vein. The echocardiographic findings were consistent with the clinical features of CP. The result of liver biopsy eventually excluded Wilson Disease. Moreover, enhanced chest CT showed thickened and calcified pericardium, as well as mild pericardial effusion, which also highly suggested CP (Figure, C).

Pericardiectomy was performed via median sternotomy without cardiopulmonary bypass. After opening the parietal pericardium carefully, we found complete separation and no adhesion between visceral and parietal pericardium, which was extremely rare (Figure, D). The thickened visceral pericardium entirely constricted the heart and compromised the diastolic filling of the heart. The visceral pericardium was dissected laterally, superiorly, and inferiorly as much as possible. To the right, visceral pericardium was dissected across the atrioventricular groove to the lateral wall of the right atrium. To the left, dissection proceeded across the ventricular septum to the lateral wall of the left ventricle. Moreover, dissection was carried superiorly onto the pulmonary trunk and inferiorly onto the diaphragm. Her central venous pressure reduced from 19 to $9 \mathrm{mmHg}$ after surgery. Pathology revealed fibroplasia and focally mild chronic inflammation and neovascularization in the visceral pericardium. The patient had an uneventful recovery and was discharged home on the 7 th postoperative day. Three years after surgery, echocardiography revealed a normal-sized left and right ventricle with good systolic and diastolic function.

\section{DISCUSSION}

Pericarditis is the most common form of pericardial disease worldwide with an incidence of 3-30 cases per 100,000 persons per year, accounting for about $0.2 \%$ of all hospital cardiovascular admission [Imazio 2015]. The common etiology of pericarditis could be infectious causes (for example tuberculosis and viral infection), noninfectious causes such as tumor, trauma and autoimmune disease, and idiopathic causes. The risk of developing CP following the first episode of acute pericarditis was evaluated in 500 patients over a mean followup time of 6 years. The results found that the overall rate of developing $\mathrm{CP}$ was about $1.8 \%$ while the nonviral/nonidiopathic causes were of the highest rate of about $8.3 \%$ [Imazio 2011]. Patients with CP will develop progressive overloading with fluid, congestion of systemic veins, and eventually right heart failure. In a series of 135 patients with CP, $67 \%$ of the patients presented with heart failure, $8 \%$ with chest pain, $6 \%$ with abdominal symptoms, $4 \%$ with atrial arrhythmia, and even 5\% with cardiac tamponade [Ling 1999]. In other study, manifestations of severe CP included $37 \%$ of patients with ascites, $53 \%$ with hepatomegaly, $35 \%$ with pleural effusion, and $76 \%$ with peripheral edema [Schwefer 2009]. These often lead to the misdiagnosis of chronic liver disease. In our case, the patient was initially suspected as having Wilson Disease, due to her manifestations of decreased serum ceruloplasmin and cirrhosis.

Two consensus studies about multi-modal imaging provide imaging and diagnostic considerations based on expert opinion, which are first-level imaging techniques of echocardiography and chest $\mathrm{x}$-ray, and second-level imaging techniques of chest CT and cardiac magnetic resonance (CMR). TTE can provide important information for the diagnosis and differentiation of $\mathrm{CP}$ from restrictive cardiomyopathy [Bao 2018]. According to Mayo Clinic Criteria of echocardiographic diagnosis of $\mathrm{CP}$, the three meaningful signs of $\mathrm{CP}$ are described as ventricular septal shift, increased levels of medial e velocity, and hepatic vein diastolic flow reversal [Welch 2014]. Chest CT is superior to CMR in detecting calcification, but CMR could assess hemodynamic events like septal bounce, pericardial inflammation, and pericardial-myocardial adherence [Verhaert 2010]. The late gadolinium enhancement of the pericardium on CMR is common and might be a predictor of reversibility following anti-inflammatory therapy [Feng 2011]. In our case, the patient presented with paradoxical interventricular septum motion and expiratory diastolic reversal in hepatic vein on TTE and calcified pericardium on CT. Given her signs, symptom, and imaging results, CP was finally diagnosed.

Classic CP is characterized by fibrous scarring and adhesion of both the visceral and parietal pericardium, leading to loss of pericardial cavity and compromised cardiac filling. The unique aspect of this case was the complete separation between visceral and parietal pericardium, which was 
extremely rare. Intraoperatively, we found the heart was entirely restricted by the thickened and calcified visceral pericardium. The parietal pericardium looked almost normal. Pericardiectomy is the only definitive treatment for $\mathrm{CP}$ with an average perioperative mortality rate of about $6 \%$ [Welch 2015]. Patients get symptom relief within 6-12 months after surgery. However, patients without features of chronic CP such as atrial fibrillation, pericardial calcification, or hepatic dysfunction, are recommended for 2-3 months of conservative medical therapy like diuretics and anti-inflammatory drugs before pericardiectomy [Laghari 2013].

\section{Conclusion}

We experienced a case of CP with rare intraoperative findings of complete separation between visceral and parietal pericardium. This is often misdiagnosed as chronic liver disease. We emphasize the importance of maintaining clinical suspicion of constrictive pericarditis in young patients with unexplained cirrhosis or features of elevated systemic venous pressure. For symptomatic CP patients without relief of symptoms after conservative therapy, pericardiectomy is highly recommended.

\section{REFERENCES}

Bao L, Huang J, Wang F, Pang L, Wang Y, Shi H. 2018. Echocardiography with elevated central venous pressure diagnosing constrictive pericarditis more accurately than chest CT scan: A retrospective study in 36 cases. Heart Surg Forum 21:E190-3.
Feng D, Glockner J, Kim K, et al. 2011. Cardiac magnetic resonance imaging pericardial late gadolinium enhancement and elevated inflammatory markers can predict the reversibility of constrictive pericarditis after antiinflammatory medical therapy: a pilot study. Circulation 124:1830-7.

Imazio M, Brucato A, Maestroni S, et al. 2011. Risk of constrictive pericarditis after acute pericarditis. Circulation 124:1270-5.

Imazio M, Gaita F, LeWinter M. 2015. Evaluation and treatment of pericarditis: A systematic review. JAMA 314:1498-1506.

Laghari AH, Tai JM. 2013. Heavily thickened pericardium with constrictive pericarditis. J Pak Med Assoc 63:639-41.

Ling LH, Oh JK, Schaff HV, et al. 1999. Constrictive pericarditis in the modern era: evolving clinical spectrum and impact on outcome after pericardiectomy. Circulation 100:1380-6.

Schwefer M, Aschenbach R, Heidemann J, Mey C, Lapp H. 2009. Constrictive pericarditis, still a diagnostic challenge: comprehensive review of clinical management. Eur J Cardiothorac Surg 36:502-10.

Verhaert D, Gabriel RS, Johnston D, Lytle BW, Desai MY, Klein AL. 2010. The role of multimodality imaging in the management of pericardial disease. Circ Cardiovasc Imaging 3:333-43.

Wei S, Zhang L, Cui H, Li L, Ren T, Jiang S. 2019. Surgery for patients with radiation-induced constrictive pericarditis. Heart Surg Forum 22:E466-9.

Welch TD, Ling LH, Espinosa RE, et al. 2014. Echocardiographic diagnosis of constrictive pericarditis: Mayo Clinic criteria. Circ Cardiovasc Imaging 7:526-34.

Welch TD, Oh JK. 2015. Constrictive pericarditis: old disease, new approaches. Curr Cardiol Rep 17:20. 